\title{
Space-Time Cubification of Artistic Shapes
}

\author{
Quentin Corker-Marin $\quad$ Valery Adzhiev Alexander Pasko \\ The National Centre for Computer Animation, Bournemouth University, UK
}
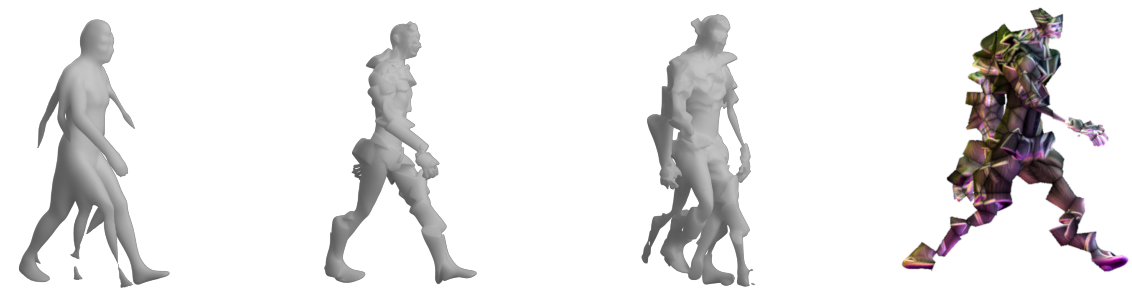

Figure 1: From left to right: 4D Cubist Camera projection of articulated human figure with space-time blending between time instances; Cubification of articulated human figure; Cubification of articulated human figure with $4 D$ Cubist camera projection; 3D Cubist camera projection with artistic effects of cubified articulated human figure.

\begin{abstract}
This poster describes an original approach to creating and producing artistic shapes in a cubist style. We propose mathematical models and algorithms of adding cubist features to (or cubification of) time-variant sculptural shapes as well as a practical technological pipeline embracing all the main phases of their production. A novel method is proposed for faceting and local distortion of the given artistic shape. A new concept of a $4 \mathrm{D}$ cubist camera is introduced for multiple projections from $4 \mathrm{D}$ space-time to 3D space and combining them using space-time blending to create animated sculptures. 3D printing for stop-motion animation is considered as one of the final pipeline processing stages. The proposed techniques are implemented with artist friendly user interfaces and experimental results are presented.
\end{abstract}

\section{CCS CONCEPTS}

- Computing methodologies $\rightarrow$ Computer graphics; Procedural animation; Shape analysis;

\section{KEYWORDS}

Hybrid Representation, Space-Time Blending, Cubism, 3D Printing

ACM Reference format:

Quentin Corker-Marin Valery Adzhiev Alexander Pasko The National Centre for Computer Animation, Bournemouth University, UK. 2017. Space-Time Cubification of Artistic Shapes. In Proceedings of SIGGRAPH 2017 Posters, Los Angeles, CA, USA, August 2017, 2 pages.

https://doi.org/10.475/123_4

\section{INTRODUCTION}

Dynamic sculptures created with help of computer-aided design and animation tools and related to a proper artistic style and tradition within a particular artistic movement are surprisingly rare. Of

*\{i7624405, vadzhiev, apasko\}@bournemouth.ac.uk

SIGGRAPH 2017 Posters, August 2017, Los Angeles, CA, USA

2017. ACM ISBN 123-4567-24-567/17/06 ...\$15.00

https://doi.org/10.475/123_4 course, there are well-established methods and tools allowing for modelling, rendering and animation of an almost infinite variety of shapes. There are also some powerful design tools for sculpturing static shapes [Séquin 2005]. However, creating dynamic art shapes are mostly concerned with traditional engineering and manufacturing practices. In this project we consider an original approach to creating artistic shapes related to the cubism movement.

This project has also served as inspiration for some original theoretical concepts integrating modelling and animation in the context of multidimensional conceptual space. There are parallels between cubism and 4D space, $\mathrm{nD}$ space and non-Euclidian geometries [Robbin 2006] which have influenced the project.

\section{OUR APPROACH}

\subsection{Overview}

Cubist painters moved away from traditional methods of projecting a 3D scene onto a 2D canvas, instead experimenting with using multiple viewpoints in a single composition. We propose to look at the theme of taking an $\mathrm{n}$ dimensional object or scene and reducing it to an n-1 dimensional representation through the new concept of a $4 \mathrm{D}$ cubist camera, which, by analogy with the well-established 3D cubist camera [Glassner 2004], is projecting two or more instances of a time-variant shape from $4 \mathrm{D}$ space-time to $3 \mathrm{D}$ space at the given time moment with possible preliminary blending between these instances. Whilst the 3D cubist camera [Arpa et al. 2012] deals with just rendering, this novel $4 \mathrm{D}$ cubist camera realises integration of modelling and animation phases through blending (i.e. compressing) a few frames generated on the basis of a faceted model with attached time-variant transformations into one thus producing cubist effect.

We propose a general shape model and develop algorithms to implement all the distinctive features of cubist sculptures. Then, provide a combination of the tools into a single system with artist's interactive or procedural control. All components of the shape model have to be time dependent to support a sculpture changing in time. We will use a hybrid representation combining polygonal 


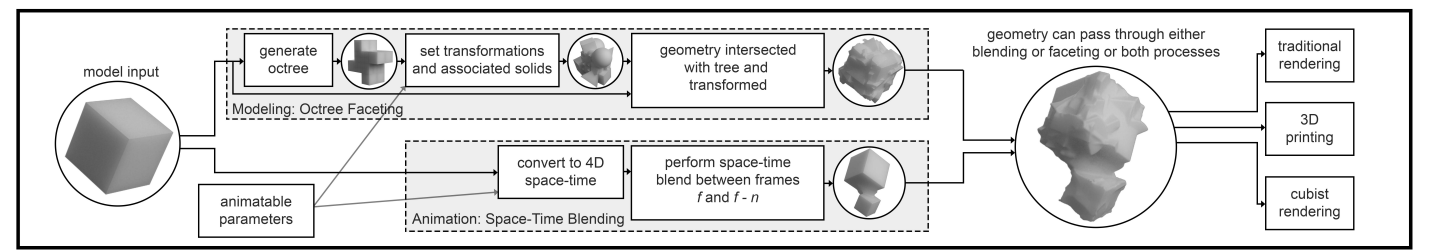

Figure 2: Cubification and animation production pipeline.

meshes with signed distance fields (SDFs). We will cover all the steps through the full production pipeline from data acquisition, to shape modelling, animation, rendering and 3D printing (Fig. 2).

\subsection{Shape Faceting}
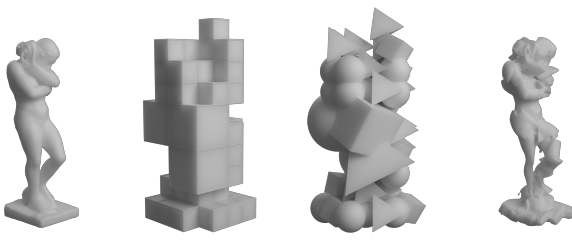

Figure 3: Steps of faceting the given sculpture from left to right: initial shape (scanned model, Three D Scans); corresponding octree; associated primitive solids; rotated and scaled facets combined with manipulated initial mesh.

Cubification of an artistic 3D shape is based on faceting using adaptive subdivision into solid pieces (facets). Octree-based subdivision is used as it provides a loose form of feature sensitivity. Shapes and transformations are then associated with each of the leaf nodes in the resulting tree structure. Each of the shapes are then intersected with the original shape and transformed using their associated transformation. The resulting facets are then combined with the initial mesh to produce the final result (Fig. 3). The transformations associated with each facet can be animated by the artist to produce dynamic sculptures.

\subsection{D Cubist Camera}

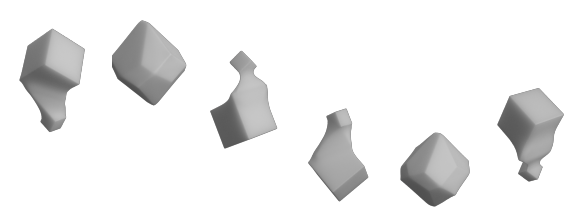

Figure 4: Space-time blending applied to non-adjacent instances of a rotating and oscillating cube.

We employ space-time blending [Pasko et al. 2004] for combining (or compressing) several shape instances (frames) into a single shape. Note that in this process animation is considered as modelling in $4 \mathrm{D}$ with projections to $3 \mathrm{D}$. Another aim of using space-time blending was to generate motion trails to follow the facets produced by the octree method described earlier. To achieve this, instead of blending between two separate objects, the blend is performed between non-adjacent shape instances (frames) $t$ and $t-n$. This allows for the effects of the surrounding frames of an animation to influence the current frame being viewed, producing a cubist-type projection of $4 \mathrm{D}$ space-time onto $3 \mathrm{D}$ space (see fig. 1 and fig. 4).

\subsection{Implementation and Experiments}

Houdini was a natural choice as the host application for the tools developed in this work. The implemented techniques rely heavily on the use of sparse volumes (discretised SDFs) from the OpenVDB library developed by DreamWorks Animation.

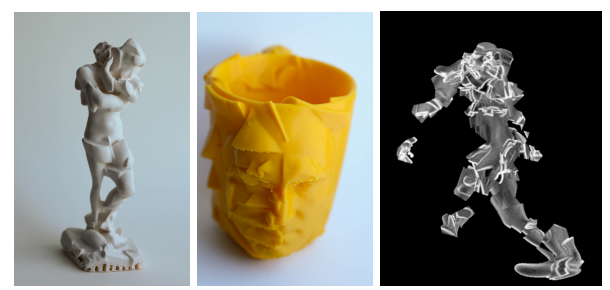

Figure 5: Left to right: $3 D$ printed results showing faceted statue; a faceted mug from 3D scan data; a faceted articulated human rendered with a $3 D$ cubist camera.

\section{CONCLUSION}

We have proposed an original approach to adaptive cubification of existing sculpture models including faceting and local distortions. A new concept of a 4D cubist camera as a projection method from $4 \mathrm{D}$ to $3 \mathrm{D}$ space was introduced. A pipeline embracing all the phases of artistic production from data acquisition, to shape modelling, animation, rendering and 3D printing has been realised. Some artefacts produced during this project can be seen in Fig. 5, the supplementary video and images.

\section{REFERENCES}

Sami Arpa, Abdullah Bulbul, Tolga Capin, and Bulent Ozguc. 2012. Perceptual 3D rendering based on principles of analytical cubism. Computers \& Graphics 36, 8 (2012), 991-1004.

Andrew Glassner. 2004. Digital cubism. IEEE Computer Graphics and Applications 24, 3 (2004), 82-90.

Galina Pasko, Alexander Pasko, and Tosiyasu Kunii. 2004. Space-time blending. Computer Animation and Virtual Worlds 15, 2 (2004), 109-121.

Tony Robbin. 2006. Shadows of Reality: The Fourth Dimension in Relativity, Cubism, and Modern Thought. Yale University Press, New Haven and London.

Carlo H Séquin. 2005. CAD tools for aesthetic engineering. Computer-Aided Design 37, 7 (2005), 737-750. 